\title{
Michon le meuble. Entretiens dans la pièce d'à côté
}

\author{
Virginie Harvey \\ Université du Québec à Montréal
}

Et si on prenait le problème à l'envers? $\mathrm{Si}$, pour aborder l'entretien, on commençait par ceux qui n'ont jamais eu lieu. Parce que s'entretenir avec un écrivain n'est pas chose aisée et que l'exercice connaît aussi ses ratés, dans la réalité comme dans la fiction. Le cas de Clément Cadou est à ce titre exemplaire. Dans Bartleby et compagnie, Enrique Vila-Matas raconte l'histoire d'un jeune homme de quinze ans, Clément Cadou, qui désire ardemment devenir écrivain. Clément est un fervent admirateur de Gombrowicz, dont il maîtrise des passages entiers de l'œuvre. La chance veut que ses parents connaissent Gombrowicz, qu'ils invitent donc un soir à dîner. 
Contre toute attente, Clément, devant Gombrowicz son modèle, demeure muet comme une carpe. Ou plutôt, comme un meuble : «Le jeune Cadou fut si impressionné de rencontrer Gombrowicz entre les quatre murs de chez ses parents qu'il ne desserra presque pas les dents de la soirée et finit par se prendre littéralement pour un meuble du salon où ils dînaient. » (2002, p. 42) Gombrowicz parti, Clément continue de faire le meuble. On raconte qu'il passa ainsi «toute sa vie», pour «oublier d'écrire», à « se prendre pour un meuble» (p. 43), balayant du coup ses rêves de littérature sous le tapis du salon. L'histoire veut aussi que, «dans l'espoir de combler le vide qu'avait creusé en lui son irrémédiable renoncement à l'écriture », il se mette à peindre. Jusqu'à sa mort précoce, Clément peint le même sujet: des meubles. Et chaque tableau porte le même titre, «énigmatique, répétitif »: autoportrait. Juste avant de mourir, on raconte encore que Clément légua à ses parents, à titre d'« œuvres complètes » (p. 44), l'épitaphe suivante: "J'ai tenté sans succès d'être d'autres meubles encore mais cela même ne m'aura pas été permis. Aussi n'ai-je été, toute ma vie, qu'un seul meuble, ce qui, après tout, n'est pas rien si l'on s'avise que tout le reste est silence. »

Entretien raté, s'il en est, entre Gombrowicz, l'écrivain modèle, et Clément, l'écrivain raté, négatif, l'interviewer tout aussi raté; entre Gombrowicz et Clément le meuble. Aussi surprenante que puisse paraître cette histoire toute "mobilière », il appert pourtant que cette représentation de l'écrivain raté en "meuble» trouve ailleurs un écho, dans les entours d'une œuvre que nous nous proposons ici d'aborder, celle de Pierre Michon. Dans la préface (« Le guéridon et le dieu bleu ») de son récent recueil d'entretiens, Le Roi vient quand il veut, Michon ouvre son texte en attribuant à nul autre que 
Victor Hugo le "meilleur recueil d'entretiens qu'[il] connaisse » (2007, p. 7). Ce recueil consiste en le Livre des Tables, procèsverbal déroutant qui témoigne des célèbres séances spirites d'Hugo durant son exil à Jersey où, pendant près de deux ans (1853-1854), il a fait «tourner les tables » dans l'objectif de les faire «parler». En partant de la préface du Roi vient quand il veut, nous chercherons à saisir plus avant ce qu'implique l'admiration de Michon pour le Livre des Tables, de quelle façon ce dernier peut tenir lieu de "modèle" pour son présent recueil et en quoi cette "technique » hugolienne de l'entretien est chez lui réinvestie en influant sur sa conception de l'invention littéraire. Pour ce faire, il s'agira de suivre la piste d'Hugo et, plus précisément, d'un Hugo «spirite » et «interviewer» - ou, du moins, les traces d'une bouche d'ombre —, de la préface à l'œuvre, puis de l'œuvre aux entretiens, en tentant de voir comment cette influence construit également des figures spécifiques de l'écrivain chez Michon.

\section{La loi des tables : le mode codé de l'éloquence}

Dans sa préface, Michon rappelle d'abord que les tables tournantes de Jersey s'exprimaient "selon un mode codé» (p. 7): «On convenait avec elles que les coups qu'elles frapperaient seraient les lettres de l'alphabet [et on écrivait] la lettre à laquelle elles s'arrêtaient. On obtenait ainsi, lettre à lettre et mot à mot, des phrases et des pages entières. » (Vacquerie, 1996, p. 7-8) Nécessaire au dialogue avec l'esprit de la table qui ne peut s'exprimer par la parole, cette méthode «codée», qu'il semble difficile de rapprocher des pratiques d'entrevue actuelles, est néanmoins reliée par Michon, non sans ironie, à une forme d'entrevue bien contemporaine: le mode 
codé est « un peu long et laborieux, mais ni plus ni moins que lorsqu'on donne une interview par e-mail. » (2007, p. 7) Par l'intermédiaire des tables et du mode codé, Hugo communique ainsi avec Chateaubriand, Shakespeare et Galilée, Napoléon-lepetit, Mahomet et Molière, mais aussi avec l'Ombre du Sépulcre, la Mort et sa petite fille, morte trop tôt. Accroché à son meuble à trois pieds, «endeuillé, exilé » (p. 8), Hugo faisait parler «les grands noms [...], les grands morts». Et cet interviewer hugolien, confirme Michon, est « un bon interviewer » (p. 7), qui "préfère s'entretenir avec des morts compétents plutôt qu'avec des imbéciles vivants » et sait poser «à chacun les questions à quoi chacun peut spécifiquement répondre».

Ce Livre des Tables, devant lequel nous ne pouvons, selon Michon, qu'être « morts de rire $^{1}$ » (p. 7), peut devenir le modèle d'un recueil d'entretiens dans la mesure où l'écrivain reconnaît dans le geste de l'interviewer Hugo une certaine « sagesse » :

Peut-être, quand le monde est idiot, est-il sage de jouer seul dans son coin la comédie du monde, faire tous les rôles et en jouir, danser seul avec les grands morts sur les ruines du monde. Il est sage d'interroger des meubles, tables ou livres, qui savent parler. Nous aussi, nous interviewons des meubles, moins éloquents que le guéridon. Nous posons à n'importe quel écrivain, c'est-à-dire à l'heure qu'il est tout le monde, les questions que Hugo posait, lui, à qui de droit, à qui pouvait lui répondre, à Jésus ou Galilée. (p. 8)

Plus encore, si l'on conçoit qu'il est « sage d'interroger des meubles qui savent parler », l'interview de l'écrivain serait dès lors un

\footnotetext{
${ }^{1}$ Michon reprend peut-être ici la pensée d'Hugo qui rapportait que le Livre des Tables, s'il venait à être publié, « serait accueilli par le sot esprit français que représente Le Charivari, et que ce livre, au lieu d'être accueilli par le respect et la foi du genre humain, serait accueilli par un immense éclat de rire ». (Adèle Hugo, 1984, p. 106)
} 
exercice absent de sagesse, une "comédie du monde » cette fois insensée puisque l'écrivain, s'il est un meuble, est "moins éloquent que le guéridon »; il est un meuble qui, ni plus ni moins, ne sait pas parler. Et c'est cette situation qui fait dire à Michon que «[n]ous nous trompons d'interlocuteur. Ceux que nous interviewons ne nous apprendront rien. » (p. 9)

D’un côté, donc, Clément le meuble, figure de l'écrivain raté, de l'entretien négatif qui, par une paralysie verbale, fait d'un devenir écrivain un mobilier, signe une «métamorphose domestique» (Vila-Matas, 2002, p. 43), signale la disparition, le silence. De l'autre, Michon le meuble, figure de l'écrivain interviewé, mais aussi de l'entretien insensé, puisqu'il y a toujours, là, erreur sur la personne, méprise sur ce mobilier qui manque d'éloquence qu'est l'écrivain. Cette méprise fait dire à Michon que, devant les questions «sur les grands sujets, la littérature, Dieu, le roman», il ne peut que s'«évader», qu'«échappe[r] à la question», «donne[r] les mauvaises réponses, celle qu'on retiendr[a] contre [lui]», «répond[re] à côté» (2007, p. 9). Si, "face à l'acte d'écriture», Michon soutient avoir recours à une «tactique contournée» (p. 59), approcher «l'écriture par des traverses, des biais, les milles ruses de la latéralité », passer son temps à "déplacer le tabouret» pour «biaise[r] la question», répondre "à côté », dans l'entretien, est plutôt la marque d'un échec à meubler adéquatement la pièce d'à côté, à être à la hauteur de la question, à être en somme aussi éloquent que le guéridon hugolien. 


\section{"Faisons un pas de plus dans ces choses profondes » (Hugo 1995, p. 365)}

La référence à Hugo dans la préface des entretiens, dont Michon annonce d'entrée de jeu l'autorité en la matière, n'a rien pour surprendre le commentateur informé de Michon : pensons à l'épisode, maintes fois répété, des premières strophes de $\mathrm{Booz}$ endormi, apprises par cœur à l'école primaire, de l'hommage rendu dans "Le Ciel est un très grand homme », qui figure dans son livre Corps du roi (2002, p. 69-102). La figure spécifique de Hugo l'interviewer, et de ses tables tournantes, trouve quant à elle son origine au sein même de l'œuvre de Michon : dans son récit Rimbaud le fils. Le narrateur y évoque les "vieux auteurs ", «les poètes en titre [...] dont le nom avait au moins une fois dans un contexte frôlé le mot génie » $(1993$, p. 37) : « [Q]uand par mésaventure ils étaient sans public à Guernesey, ils convoquaient par la voie des airs Shakespeare et Mozart, Virgile, qui paternellement accourus sur la mer les réassuraient [...] : et le Vieux, penché sur sa table tournante, dans son île grise était à la première d'Hernani avec le gilet rouge. » Plus loin, le narrateur en appelle encore au « Vieux en conversation avec Shakespeare » (p. 82), ajoute au final que « Hugo dans son île n'est là pour personne, il est penché, il écoute les quatre pieds de sa table taper du pied Shakespeare » (p. 51).

Il y aurait donc là une première apparition d'un Hugo en conversation, penché sur sa table. Aussi faut-il noter que la préface du Roi vient quand il veut est plus précise quant à la nature et à la provenance de la table tournante d'Hugo, laquelle est « un petit guéridon à trois pieds acheté à Saint-Hélier dans 
un magasin de jouets d'enfants» (2007, p. 7). Une caractérisation de la table-trépied, accentuée par l'usage des italiques et amenée non sans quelques «traits de mauvaise foi » (quatrième de couverture), qui rappelle que sa fonctionnalité première est du côté de l'amusement, que son usage appartient d'abord à l'enfance : la table parlante du Vieux est nul autre qu'un « jouet d'enfants », servant pourtant aux grands, au vieil interviewer Hugo, à joindre les grands morts². Cette idée du jeu d'enfants, dans tout ce que ce dernier implique comme mise en scène, théâtralisation du monde ou plaisir enfantin, est en somme aussi ce qui participe au «modèle» du recueil d'entretien michonnien, où pour élaborer de "grands" discours, il est peut-être sage de faire le « petit », « sage de jouer seul dans son coin la comédie du monde, faire tous les rôles et en jouir» (p. 8 : nous soulignons).

Les conversations de Hugo avec les grands absents sont aussi, dans Rimbaud le fils, un prétexte pour aborder cette idée de "génie », dont le narrateur dit qu'elle a tout à voir avec « le Verbe qui depuis le début souffle où il veut et n'a pas de résidence, Charleville ni Patmos, ni Guernesey » (1993, p. 37), avec cette "révélation d'En-Haut, [...] cet attribut comme surnaturel qui ne se manifeste jamais en soi [...] mais [...] dans d'infimes effets, et qu'on vérifie dans la perfection de petits

\footnotetext{
2 Il faut aussi ajouter qu'entre les entretiens de Hugo dans Rimbaud et ceux de la préface du Roi vient quand il veut, il appert que leur lieu s'est déplacé, de l'île de Guernesey à celle de Jersey, et que la table-trépied a magiquement perdu un pied... En effet, alors que le meuble de Hugo dans Rimbaud possède quatre pieds, il en compte seulement trois dans la version rejouée de la préface. Si la lecture du Livre des Tables confirme ce dernier compte, on peut avancer que Michon a profité de l'écriture de la préface pour relire le Livre des Tables de Hugo, rétablissant par là les supports du meuble et rectifiant la scène originelle, qui a bien eu lieu à Jersey et non à Guernesey.
} 
morceaux de langue codée plus ou moins longs, écrits noirs sur blanc » (p. 35). Cette «langue codée » n'est pas sans rappeler celle qui émane de la table tournante d'Hugo, alors qu' «aux questions hugoliennes, le guéridon répond en tapant du pied, selon un mode codé » (2007, p.7), tel que le précise Michon dans la préface de ses entretiens.

Il faudra en tous cas attendre six ans plus tard pour que Hugo l'interviewer revienne hanter le discours de Michon, dans un espace qui n'est pas encore celui de la préface mais qui appartient déjà à l'entretien, lequel sera éventuellement repris dans Le Roi vient quand il veut. En 1997, dans une entrevue que donne Michon pour la revue Recueil, Tristan Hordé le questionne alors sur la bâtardise, sur l'absence du père, qu'il dit « centrale » dans son œuvre. Michon répond, à propos du père :

Je ne l'ai pas connu, ou alors énorme comme le Saturne de Goya, quand il se penchait sur mon berceau. Je ne crois pas m'en souvenir. Je porte son nom dans un rêve. Il n'existe guère. C'est un personnage de légende, un deus absconditus, une troisième personne idéalement absentée. En tant que tel il est le dédicataire noir des Vies minuscules ; il est sûrement la grande figure effondrée dont mes histoires sont veuves, Alaric dans L'Empereur d'Occident, Vélasquez dans le Goya et saint Martin dans le Lorentino. Peut-être même que c'est lui, le Vieux toqué penché sur sa table tournante, Hugo [...]. (2007, p. 39)

Hugo l'interviewer, le «Vieux toqué penché sur sa table tournante», serait donc, en plus d'une incarnation du génie littéraire, une figure possible du père, tout comme cette autre figure penchée, énorme comme le Saturne de Goya, qui «se penchait sur [le] berceau » du jeune Michon. Deux figures du père penché, de pères qui se penchent sur un meuble, berceau ou table tournante. La première figure, dévorante: c'est le Saturne de Goya, qui mange son enfant pour éviter que 
n'advienne la malédiction qui le verrait détrôné par sa progéniture. L'autre figure, vieille et toquée, celle d'Hugo le spirite, l'interviewer déjanté, incarnation de cette "troisième personne idéalement absentée » qu'est le père, le sien et celui de tous, penché sur sa table tournante, tentant de conjurer l'absence que pourtant il incarne, de faire sortir de son silence, par la folle fréquentation d'une table, quelque absent. La folie qui pousse l'écrivain à avoir commerce avec les meubles est bien celle qu'évoque le narrateur de Rimbaud le fils lorsqu'il raconte le moment où Rimbaud «s'aperçoit que la poésie ça descend, [...] [que] c'est une pente dégringolée à vide qui vous amène dans un hôtel de Bruxelles - ou à Guernesey devant des tables tournantes, souverain, magique, charlatan : la pente va à Guernesey, avec beaucoup de chance » (1993, p. 89).

Ainsi, pour Michon, être interviewé, c'est faire le meuble, incarner celui qui n'a fondamentalement pas la réponse à la question. Il est en cela similaire aux êtres de l'ombre qui peuplent l'œuvre hugolienne et qui n'arrivent jamais à assouvir la soif de savoir du poète en quête existentielle. Par ailleurs, produire un recueil d'entretiens, c'est pour Michon s'inscrire par suite dans cette lignée de Hugo, dans la lignée de figures possibles de pères penchés sur le meuble, de l'entretien "toqué », qui branche le poète sur le génie des choses. Car le poète est lui aussi une figure penchée sur un meuble, tel que l'attestent les nombreuses apparitions, dans Rimbaud, d'écrivains "penchés sur leur bureau de poète »: Rimbaud et Verlaine, qui «bien sagement studieux de part et d'autre d'un même bureau de poète dans Londres [...] restaient penchés » (1993, p.66); Banville qui, prenant la forme du Gilles de Watteau, « ouvre la ronde des lecteurs de Rimbaud [...], penché [lui aussi] sur [son] bureau de poète» (p.51); puis, plus 
généralement, les commentateurs de Rimbaud, qui " penchés à leur bureau de poète [...] en silence [...] nous entretiennent de lui » (p. 55). C'est encore le photographe Carjat qui s'apprête à immortaliser Rimbaud et qui "penché sur lui l'observe» (p. 89). C'est «nous », qui « lisons et remâchons cette lettre [du Voyant], penchés à nos bureaux de poète [...] [,] nous [qui] y répondons » (p. 61-62). Puis c'est Michon, qui, « [p]enché pardessus [n]otre épaule dans la bibliothèque de Confolens", regarde des portraits d'auteurs canoniques, « avec [n] os yeux » (p. 99).

Par ailleurs, si on se reporte à l'œuvre de Hugo, et spécifiquement aux textes qui sont en relation avec des bouches d'ombre, cette idée de poète penché se trouve aussi développée. Dans un fragment de Dieu, par exemple, "[l]es Lucrèces, les Jobs, les blêmes Jérémies, / La lèvre émue encor de leurs strophes frémies, / [Sont] [c]ourbés sous l'épouvante [...] / Tous ces grands effarés porteurs des harpes sombres ${ }^{3}$. » Plus loin, on lit encore: "Allons, mage! fais-toi reconnaître du gouffre! / N'es-tu pas le voyant dont la prunelle luit? / [...] Toutes les vérités / Sont dans ce fauve amas d'ombre et d'obscurités. / Travaille, penche-toi ; sache les en extraire ${ }^{4}$. »

Chez Hugo, le poète-mage se penche ainsi en signe d'effort : soit il se courbe sous l'épouvante de l'ombre pour faire vibrer sa harpe sombre, soit il se penche pour "travailler", pour extraire les vérités de l'ombre. Cela fait écho à l'action de se pencher sur un meuble, bureau de poète, berceau, table tournante, à trois ou quatre pieds que l'on trouve chez Michon et qui serait un geste commun à l'écrivain, au poète, aux toqués

\footnotetext{
3 Victor Hugo, Dieu, fragments, section I, fragment 35a [1856].

4 Ibid., fragment 466b [1856-1858].
} 
et aux pères dévorateurs; à celui qui écrit dans le silence et aussi à celui qui, dans le grand «tapage » (p.62) d'une table, interroge l'absence.

Geste répété de l'évocation de la figure de Hugo l'interviewer, que pose Michon trois fois plutôt qu'une, dans l'œuvre, puis dans l'entretien, puis dans la préface d'un recueil d'entretiens. Lentement la figure de Hugo investit les bords, part du centre et se déplace vers les seuils; c'est là que mène peut-être la pente descendante de Guernesey, « avec beaucoup de chance ».

\section{"Ya-t-il quelqu'un?5 » : la littérature comme entretien}

Nous revoici donc au seuil. Par le biais du modèle de l'entretien hugolien dans la préface du Roi vient quand il veut, de cette figure construite, à la fois dans l'œuvre et dans ses entours, Michon interroge simultanément le statut de l'écrivain, de la littérature et de l'interview, ce qui n'est pas sans nous informer sur sa conception de l'invention littéraire. Si, par l'entretien, nous conversons avec des meubles qui ne nous apprendront rien, entreprise aussi insensée que celle de l'interviewer toqué penché sur sa table, l'étude des entretiens montre plus encore que la littérature en général se conçoit également chez Michon comme une "tentative de communication» qui ne peut que passer par l'absence, que par une tierce partie. Dans le numéro de mai 2009 du Matricule des anges, dont il fait la couverture, l'auteur à ce sujet affirme : « J'envisage la littérature comme un

\footnotetext{
5 Question posée par Hugo lors d'une séance spirite à Jersey, le vendredi 29 septembre 1854, alors qu'il s'entretenait avec «La Mort» et que la table s'arrêta brusquement, pour ensuite relever tout aussi brusquement le pied (Vacquerie, 1996, p. 194).
} 
essai pour se mettre en communication avec le genre humain mais en passant par un tiers absent qui est le divin. » (p. 26) Pour se penser, la littérature aurait donc besoin de cette tierce partie absente, serait élaborée comme une forme d'entretien avec elle, rappelant du coup l'imaginaire de la bouche d'ombre hugolienne, le «Causons» (1995, p. 363) que lance le spectre au poète dans «Ce que dit la bouche d'ombre», autant que le «Écoute bien » (p. 362).

Cette idée du «tiers absent », elle était déjà contenue chez Michon dans des entretiens antérieurs, où il soutenait que « [1]a littérature est une forme déchue de la prière, la prière d'un monde sans Dieu. On écrit comme jadis on s'adressait à quelqu'un, à un autre qu'autrui, à une grande instance fantasmatique mais comblante, qu'on appelait Dieu. Le grand Tiers. » (2007, p. 29) Il ajoutait encore: «[I]l est sûr que j'appelle une sorte de transcendance indéfinissable. [...] [Attirer Dieu], [t]outes proportions gardées, je ne crois pas tenter autre chose. » (p. 58) Et une fois de plus, Michon ne pourra manquer de se référer à Hugo, et cette fois dans un entretien de 2009, poussant à bout notre hypothèse :

[L]e langage est la communication aussi avec les grandes puissances. Les grands délires hugoliens de la bouche d'ombre, c'est quelque chose dont je suis très près. Cette communication avec les grandes puissances. Avec les morts bien sûr, puisqu'ils sont dans l'au-delà de l'humain comme les puissances. (p. 26)

Cette adresse au "grand Tiers», aux "grandes puissances», produit corollairement dans les entretiens deux figures de l'écrivain, celle du prêtre, dont l'artiste prend la place en se «branch[ant] sur le tiers absent » (p. 28), et celle de l'oracle. Au sujet de cette dernière figure, Michon déclare que "[1]es écrivains, peut-être particulièrement aujourd'hui, sont des 
espèces de monades vaticinantes, isolés chacun sur leur pente qu'ils dégringolent comme ils peuvent » (2007, p. 157). Sorte de substance première, prophétique, que serait donc l'écrivain contemporain, que l'on trouve ici pareillement isolé sur une pente dégringolante, tout aussi « dégringolée à vide » (1993, p. 89) que celle de Guernesey dans Rimbaud le fils, sauf qu'ici, Michon se garde bien de dire où elle mène; c'est qu'il est luimême sur cette pente, Michon, et n'est pas encore à destination. Parce qu'arriver à se brancher sur le grand Tiers, réussir à entrer en communication avec les grandes puissances, ce n'est pas chose aisée, aussi vaticinant et sacerdotal que soit l'écrivain.

Les figures de prêtre et d'oracle de Michon rappellent également Hugo, pour qui «[l]e génie inspiré d'en haut qui guide les hommes s'appelle bien [...] Poète, mais aussi "voyant", "prêtre", "pontife", "prophète", "mage", "célébrateur", "révélateur", toutes désignations qui évoquent sa familiarité avec le surnaturel et le sacré » (Bénichou, 1988, p. 492). Chez Hugo, il y a tout à la fois des tentatives répétées de se mettre en communication avec la bouche d'ombre et la volonté d'être cette bouche. À propos des tables tournantes, Hugo affirme: "Quoi que la crédulité en ait dit ou pensé, ce phénomène des trépieds et des tables est sans rapport aucun [...] avec l'inspiration des poètes, inspiration toute directe. La sibylle a un trépied, le poète non. Le poète est lui-même trépied. Il est le trépied de Dieu.» Et Hugo poursuit: «Dieu n'a pas fait ce merveilleux alambic de l'idée, le cerveau de l'homme, pour ne point s'en servir. Le génie a tout ce qu'il lui faut dans son cerveau. Toute pensée passe par là. [...] La pensée est la résultante de l'homme.» (1867, p. 30) Comme le remarque Bénichou dans son ouvrage Les mages romantiques, «le poète 
est [donc chez Hugo] à la fois trépied de Dieu et cerveau humain agissant par sa propre nature » (1988, p. 520). Il s'agirait donc être une bouche et se pencher, en somme.

Comme Hugo, pour qui l'exercice des tables était loin de se limiter à "l'écoute passive d'une révélation» (p.512) Bénichou rappelle d'ailleurs l'insistance avec laquelle Hugo répétait «qu'il a[vait] déjà, ici ou là, pensé, écrit ou soupçonné ce que les tables disent »-, Michon est précisément poussé à prendre la parole parce que cette connaissance du fonds des choses lui semble impossible à atteindre. Dans l'œuvre de Michon, le constat répété d'un échec à atteindre la source des choses - Dieu, comme le père, s'y fait toujours figure absentée - provoque l'impératif d'un passage à l'écriture, d'une invention 6 . En entretien, Michon s'exprime à ce sujet par le biais de l'exemple d'une peinture de Velasquez, dont il commente le fond noir :

Vous me direz qu'il n'y a rien, là, dans ce fond noir. Eh bien non, il n'y a rien. [...] [C]'est comme le fond noir de Velasquez, l'énonciation. Ça n'est rien, mais c'est un rien violent et volontaire qui porte les figures, qui les fait tenir. Sans ce noir qui souffle au fond, il n'y aurait pas de figures. D'ailleurs est-ce qu'il y en a? C'est bien parce qu'il n'y a pas de figures, pas de pensée, pas de contenu, que l'homme de littérature est bien obligé de prendre la parole avec violence. Pas de sujet, pas de thème, pas de pensée, rien que de la volonté violente de dire, qui fait par miracle quelque chose avec rien, qui fait une forme dans laquelle s'installe, en plus, du sens. (2007, p. 140).

Michon rejoint en cela Hugo, pour qui c'est « la petitesse [de l'homme] en face de l'Infini [...] [qui] crée [...] [le] [...] droit d'interrogation et de quête » (Michon, 2007, p. 140) « du PoèteMissionnaire en quête de vérité ».

6 Au sujet du rapport de Michon au vide originel, voir Harvey (2009). 
C'est ici, en dernière instance, que le titre du recueil de Michon peut prendre tout son sens. Dire que Le Roi vient quand il veut, malgré une confusion qui semble généralisée chez les commentateurs, n'appartient pas à la logique binaire des deux corps du roi que développe Michon dans l'ouvrage du même nom et qui stipule, à la suite de Kantorowicz, que le roi a deux corps, l'un « éternel, dynastique, que le texte intronise et sacre » et l'autre «mortel, fonctionnel, relatif» (2002, p. 13). Pour le comprendre, il faut aller à la rencontre du titre, qui trouve son origine à même les entretiens, dans une réponse que donne Michon à Daniel Nadaud pour le compte de la revue de l'École des beaux-arts de Nantes, dix ans avant la publication de Corps $d u$ roi. Interrogé sur sa «quête de portraits» et sur la possibilité qu'il soit toujours plus ou moins «l'objet détourné de ceux-ci » (2007, p. 67), Michon déclare ceci, qui mérite d'être cité assez longuement :

Sans doute, mes portraits sont des autoportraits, mais à la façon dont Le Comte de Floridablanca de Goya est un autoportrait. Dans ce tableau, [...] on voit au milieu dans un flot de lumière le glorieux comte en habit écarlate, derrière le comte un sous-fifre dans l'ombre avec à la main un crayon ou un compas, et sur le côté au premier plan, tout petit et noir, Goya en personne qui montre au comte son tableau (celui donc que nous regardons) - et le comte bien sûr ne regarde pas le tableau, mais droit devant lui nous-mêmes, ou la gloire. Pardessus tout cela, au mur, de même taille que les trois hommes de chair, un portrait peint du roi. Il y a entre ces quatre personnages (les trois hommes présents et le roi peint) une circulation intense de regards, je dirais presque d'identités. Où est Goya, où s'est-il vraiment représenté? Est-il le vrai Goya de chair, bien humble et courtisan, qui tient le tableau? Est-il le beau comte chamarré qu'il rêve d'être? Ou le roi, qui est toujours en quelque sorte personne? Ou encore le type derrière, le gratte-papier absorbé par l'ombre, que Goya craint d'être, qu'il sait bien être aussi en quelque façon? Ainsi, dans ce 
que tu appelles mes portraits, je suis le sujet du portrait, le comte [...]; je suis celui qui peint, et aussi celui qui raconte, le témoin, l'humble narrateur [...]; et je suis enfin une troisième voix qui apparaît çà et là dans mes textes, qui est moi sans doute, l'écrivain, le gratte-papier qui est mangé par l'ombre, tout au fond du tableau. J'aimerais bien qu'il y ait en plus le roi, c'est-à-dire la littérature, ou le sens, ou le vrai, ou peut-être tout simplement le lecteur. Mais le roi vient quand il veut. (2007, p. 66.)

Ainsi, dire que Le Roi vient quand il veut et choisir ce bout de phrase pour titre de son recueil d'entretiens, c'est pour Michon marquer autrement cette tentative répétée pour s'entretenir avec les grandes puissances, pour se brancher sur la littérature, le sens ou le vrai, pour accrocher au mur l'apparition d'un roi, dont on prétend qu'il «est toujours en quelque sorte personne » et dans lequel peut «avec beaucoup de chance» tenir le grand Tiers absent. Dire que Le Roi vient quand il veut, c'est arriver au constat que, malgré les essais réitérés de l'écrivain "pour se mettre en communication avec le genre humain », malgré les multiples prières adressées à " un autre qu'autrui », les oracles incongrus qui font rêver d'un lecteur, qui font s'inscrire sous l'égide d'un Vieux père toqué, rien dans l'exercice de l'écriture comme de l'entretien n'est garanti. Car, parfois, le meuble ne nous apprend rien, quand l'espace d'un instant, le roi n'est pas Michon mais celui qu'il tente de joindre, penché sur sa table. Quand celui qu'il tente de joindre vient quand il veut. Mais c'est précisément cela qui fait écrire, dans la pièce d'à côté. 


\section{Bibliographie}

BÉNICHOU, Paul (1988). Les Mages romantiques, Paris, Gallimard.

HARVEY, Virginie (2009). « Fausse dent, caverne vide, trafic de dieux. La mémoire préhistorique chez Pierre Michon », dans Jean-François Hamel et Virginie Harvey (dir.), Le Temps contemporain : maintenant la littérature, Montréal, Figura, coll. « Figura », no 21, p. 37-49.

Hugo, Adèle (1984 [1852-1855]). Journal d'Adèle Hugo, Frances Vernor Guille (dir.), Paris-Caen, Lettres modernes Minard, coll. « Bibliothèque introuvable », t. III.

Hugo, Victor (1995 [1855]). « Ce que dit la bouche d'ombre », Les Contemplations, Paris, Flammarion.

- (1867). William Shakespeare, Paris, Librairie internationale.

Michon, Pierre (2009). "Fabrique de légendes», entretien avec Thierry Guichard, Le Matricule des anges, $\mathrm{n} \times 103$, mai.

- (2007). Le Roi vient quand il veut. Propos sur la littérature, textes réunis et édités par Agnès Castiglione avec la participation de Pierre-Marc de Biasi, Paris, Albin Michel.

— (2002). Corps du roi, Lagrasse, Verdier.

- (1993 [1991]). Rimbaud le fils, Paris, Gallimard, coll. «Folio ». 
VACQUERIE, Auguste (1996 [1923]). Chez Victor Hugo. Les Tables tournantes de Jersey, Paris, L'école des loisirs.

VILA-MATAS, Enrique (2002 [2000]). Bartleby et compagnie, traduit de l'espagnol (castillan) par Éric Beaumatin, Paris, Christian Bourgois.

\title{
Résumé
}

À partir de la préface du recueil d'entretiens Le Roi vient quand il veut de Pierre Michon, cet article cherche à saisir l'admiration de Michon pour le Livre des Tables de Hugo, la façon dont ce dernier peut tenir lieu de " modèle » pour son présent recueil et en quoi cette « technique » hugolienne de l'entretien est chez lui réinvestie dans sa conception de l'invention littéraire. La piste d'Hugo est suivie, celle d'un Hugo "spirite » et "interviewer ", de la préface à l'œuvre, puis de l'œuvre aux entretiens, et montre comment cette influence construit des figures spécifiques de l'écrivain.

\begin{abstract}
Based on the preface of Le Roi vient quand il veut, a collection of Pierre Michon's interviews, this article tries to understand Michon's admiration for Victor Hugo's Livre des Tables, how it can serve as a "model" for this collection and how Hugo's "technique" of the interview is reinvested in Michon's conception of literary invention. From the preface to the work, then from the work to the interviews, Hugo's track, as a "spiritualist" and as an "interviewer", is followed in order to show how this influence builds specific figures of the writer.
\end{abstract}

